\title{
应用极化水分子模型研究单分子层受限水的介电性质
}

\author{
范勤梁洪涛许贤祺吕松泰梁尊杨洋* \\ (华东师范大学 物理与电子科学学院 凝聚态物理研究所 上海 200241)
}

\begin{abstract}
摘要 受限条件下水的介电性质因测量极具挑战, 其在诸多电化学过程与反应输运过程中如何扮演关键角色从未被定 量地澄清. 本工作利用平衡态分子动力学模拟和受限体系介电性质计算方法, 系统性地探索了 $0.65 \mathrm{~nm}$ 限域尺寸、 $5 \times$ $10^{8} \mathrm{~Pa}$ 限域压强、不同温度条件下单分子受限冰和受限水的介电性质. 详细比较了恒定偶极矩 SPC/E 水分子模型和可 极化的 SWM4-NDP 水分子模型在描述受限冰、水结构与介电性质上的优劣势，包括统计分析 SWM4-NDP 模型模拟的 单分子层受限水和受限冰的瞬时分子偶极矩概率密度分布, 计算每个模拟体系的静态结构因子、静态偶极空间关联函 数、静态介电常数、体系偶极时间关联函数和德拜弛豫时间. 首次发现了极化水分子模型描述的低维度受限水和受限 冰的奇异分子极性变化, 并观察到两种模型描述静态结构性质的效果相当, SWM4-NDP 模型对于静态介电常数描述的 优势会因受限条件的增强而被大幅削减. 但在受限水介电极化弛豫动力学性质描述上 SWM4-NDP 模型明显优于 SPC/E 模型. 我们推断 SWM4-NDP 模型在探索受限水结构相变动力学以及受限体系离子输运和溶剂化动力学等过程 的模拟研究中是比 SPC/E 模型更好的选择. 本工作将在进一步开展基于受限水系统储能、传感、输运的设计工作中提 供一定的理论指导意义.
\end{abstract}

关键词 受限水; 方冰; 极化水模型; 分子动力学; 介电常数

\section{Study of the Dielectric Property of Monolayer Confined Water Using A Polarizable Model}

\author{
Fan, Qin Liang, Hongtao $\quad \mathrm{Xu}$, Xianqi $\quad$ Lv, Songtai $\quad$ Liang, Zun $\quad$ Yang, Yang* \\ (School of Physics and Electronic Science, East China Normal University, Shanghai 200241, China)
}

Abstract The direct measurement of the dielectric properties of the confined water is exceedingly challenging, result in the lack of a quantitative understanding of its critical roles in electrochemistry, interfacial reactivity and transport thermodynamics. In this paper, we employ the equilibrium molecular dynamics simulation and the linear response theory-based analytical expressions for the local permittivity tensor, to calculate the static and dynamic dielectric response properties of the monolayer ice and water confined in the $0.65 \mathrm{~nm}$ size hydrophobic slab pore under $5 \times 10^{8} \mathrm{~Pa}$ lateral pressure and different temperatures. We carry out a detailed comparative study on the performance of predicting the confined structure and dielectric response properties between two well known water molecule models, i.e., constant dipole moment SPC/E model and polarizable SWM4-NDP water model. We have analyzed the probability distributions of the instantaneous SWM4-NDP water molecular dipole moments and calculated the static structure factor, radial dipole-dipole correlation function, static dielectric tensor, total dipole autocorrelation function and Debye relaxation time of each simulation system. For the first time, we found the novel variation of the water molecular polarities, in the monolayer confined liquid and solid phase of water, due to the extreme confinement condition. The performance in describing the structural properties are found comparable between the two water models, and the enhancement of the confinement weakens the advantage of the SWM4-NDP model in predicting the static dielectric property. However, in the prediction of the dynamic properties such as dielectric relaxation time, SWM4-NDP water model is superior to the SPC/E model. Therefore, we suggest that using SWM4-NDP model in the future investigation of the structural phase transition kinetics, ionic transportation and solvation kinetics would be the better choice. The current achievement of the fundamental insight and computational data could potentially facilitate the theoretical advancements in designing new devices of energy storage, sensor, and medicine delivery based on confined water systems.

Keywords confined water; square ice; polarizable water model; molecular dynamics; dielectric property

\section{1 引言}

微纳尺度的受限水体系中, 受限水大面积接触受限
腔内壁，受到腔壁势场影响发生强弱程度不同的电极 化 ${ }^{[1]}$,与此同时水分子的排列受到空间约束和腔壁微观 结构势场调制，致使受限水体系呈现丰富多彩的有序度

*E-mail: yyang@phy.ecnu.edu.cn

Received March 4, 2020; published May 23, 2020.

Project supported by the National Natural Science Foundation of China (Nos. 11504110, 11874147), the Fundamental Research Funds for the Central Universities and East China Normal University Multifunctional Platform for Innovation (001).

项目受国家自然科学基金(Nos. 11504110, 11874147)、中央高校基本科研业务费专项资金和华东师范大学公共创新服务平台(001)资助. 
和物相结构 ${ }^{[2]}$. 界面水分子局域极化能力也影响着局域 水分子之间、水分子和其它带电粒子之间的相互作用, 进而影响诸多重要的界面物理与化学过程 ${ }^{[3 \sim 5]}$, 如表面 水化、离子溶剂化、分子输运、颗粒自组装等. 受限水 系统兼具界面水和受限物相的物理特性, 毫无疑问其介 电性质的探索具有重要的科学意义和应用价值, 近年来 也已经获得越来越多的科研关注. 例如, 受限水分子在 其平动与转动自由度部分缺失、周边电场环境的状态下 如何影响静态与动态介电性质, 至今尚未有定量的认识. 掌握受限水介电性质的变化规律, 将帮助微纳尺度药物 输运技术和高效海水淡化盐离子技术取得进步 ${ }^{[6,7]}$, 也 将促进新型纳米储能与传感器件设计 ${ }^{[8,9]}$ 领域的发展.

界面水分子分层有序伴随着电极化 ${ }^{[10]}$, 施加电场 难以进一步驱使已极化界面水分子转动作出线性介电 响应, 因此实验测量介电常数极具挑战. 近二十年来受 限水介电性质的研究集中在理论与计算模拟领域 ${ }^{[11 ~ 14]}$. 例如, Ballenegger 和 Hansen ${ }^{[11]}$ 基于线性响应理论推导出 受限腔体极性分子液体介电常数空间分布函数的理论 表达式; Galli ${ }^{[13]}$ 和 $\mathrm{Netz}^{[14]}$ 小组基于其开展的分子模拟研 究, 分别报道了受限水介电常数张量和介电弛豫性质的 强烈各向异性; Bonthuis 等 ${ }^{[12]}$ 和 Schlaich 等 ${ }^{[14]}$ 计算了受 限腔壁亲疏水性和柔性腔壁对受限水介电常数的影响 等. 2018 年, Geim 团队 ${ }^{[15]}$ 在受限水介电性质的测量方法 上取得突破, 他们利用先进的微纳加工技术结合扫描介 电显微镜与原子力显微镜, 首次精密测量了受限尺寸小 至 $0.8 \mathrm{~nm}$ 的受限液相水的介电常数. Geim 等 ${ }^{[15]}$ 提出, 对 受限水的精密实验测量结果, 将对计算模拟研究提出更 高的要求. 这是因为已有受限水介电性质的分子模拟研 究大多应用恒定偶极矩的简单水分子模型, 并不考虑水 分子极性的变化. 而实际受限水的分子极性、介电性质 以及限域条件之间的物理关联都亟待探究.

本工作的另一个研究动机涉及受限水的固-液结构 相变. Algara-Siller 等 ${ }^{[16]}$ 运用透射电镜直接观测到了范 德瓦尔斯作用下两片石墨烯中二维正方晶格的受限方 冰, 并监测到电子束移动可以驱动受限方冰的无序化. 这个实验发现意味着应用外场控制限域水不同物相间 结构相变的可能性, 及利用受限冰相变定量调控介电储 能性质的潜在应用价值. 虽然上文介绍了受限水介电性 质的实验测量技术上的突破, 但目前仍无法做到同时定 量控制结构相变和定量测量介电性质. 本文作者已陆续 开发了低维固-液界线的平衡态分子动力学模拟技术, 模拟了单分子层受限冰-水(固-液)两相平衡 ${ }^{[17]}$, 并预言 了二维方冰晶界的预熔化相变液膜宽度的定量物理规 律 ${ }^{[18]}$. 在这些模拟研究基础上, 近一步精确计算单分子 层受限冰和受限水的介电性质, 对于评估受限冰相变介 电器件应用的可行性至关重要.

因此本工作的目标是计算单分子层受限水和受限 冰体系的介电性质. 此前, 受限水的模拟研究大多使用
经典恒定偶极矩刚性水分子模型，以牺牲分子极性描述 精度换取计算速度优势. 但当关注的问题涉及介电性质 时, 恒定偶极矩刚性水分子模型不再适用，需要使用极 化水分子模型以动态适应外界电场环境变化 ${ }^{[19]}$, 从而 更加准确地预测介电常数. 到目前为止, 尚未有研究系 统比较过简单的恒定偶极矩水分子模型和极化水分子 模型在描述受限水体系介电性质的具体优劣表现.

基于上述研究背景和研究目标, 本论文将利用平衡 态分子动力学模拟和受限体系介电性质计算方法, 详细 比较两种水分子模型(恒定偶极矩模型和极化模型)在描 述受限单分子层液相水和固相冰的介电性质上的优劣 势. 我们分别用两种水分子模型模拟了在 $0.65 \mathrm{~nm}$ 限域 尺寸、 $5 \times 10^{8} \mathrm{~Pa}$ 限域压强条件下的四个不同温度的平 衡态单分子层水和冰. 计算了每个模拟体系的静态结构 因子、静态偶极空间关联函数、静态介电常数、体系偶 极时间关联函数和德拜弛豫时间. 另外, 我们统计了极 化水模型描述单分子层受限水和受限冰的水分子偶极 矩量值概率密度分布. 得到了关于极化水模型在描述受 限水/冰体系介电性质具体优势较为全面系统的认识, 并首次发现了单分子层受限水、冰的分子极性区别于块 体系统的奇异特性. 本工作获得的计算结果能够为受限 水领域研究提供重要数据, 对进一步开展基于受限水系 统储能、传感、输运的理论设计具有重要指导意义.

\section{2 计算方法}

\section{1 水分子模型}

采用两种水分子模型开展本论文中的模拟与计算 工作. 一种是最常用的 $\mathrm{SPC} / \mathrm{E}$ 水模型 ${ }^{[20]}$ (具体作用势参 数参考引文 ${ }^{[17]}$. 另一种是 2006 年 Lamoureux 等开发的 Drude 极化水模型 ${ }^{[21]}$ (全称 SWM4-NDP, 4-site simple water model with new drude polarizability). 如图 1 所示, Drude 极化水模型中引入一个假想的极小质量带电荷粒 子. 这个粒子通过一个假想的弹簧连接着氧原子粒子, 带电粒子的电荷、弹性振荡的瞬时位置、其所在空间点 位的电场强度与自身极化率决定了水分子的动态诱导 偶极矩数值.

除了 Drude 极化水分子模型之外还有两套著名的极 化水分子模型, 分别是诱导点偶极水分子模型 ${ }^{[22]}$ 和基 于电负性平衡原理的涨落电荷水分子模型 ${ }^{[23]}$. 这两套 模型, 前者相较于 Drude 模型缺点在于矢量化长程库伦 能力场的修改过于繁杂, 后者相较于 Drude 模型的缺点 在于极化率张量描述能力的欠缺(如线性分子只能够获 得轴向的极性). Drude 模型很好弥补了这些欠缺, 在计 算效率上有了显著提高. 除此之外, Drude 极化水分子 模型能够成功再现常温常压条件下液相块体水的静态 介电常数(见表 1)、蒸发焓、密度、自扩散系数、剪切 粘度和水化自由能等一系列关键热力学性质. 需要指出 


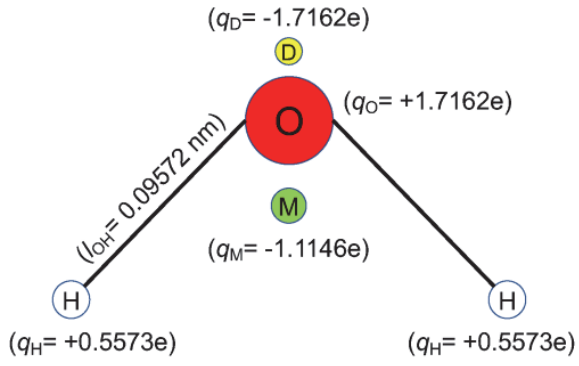

图 1 Drude 水模型结构及参数示意图. $\mathrm{D}$ 代表 drude 振荡粒子, $\mathrm{M}$ 代 表模型中刚性虚拟无质量电荷点. 键长为 $0.09572 \mathrm{~nm}, \mathrm{HOH}$ 键角为 $104.52^{\circ}$

Figure 1 The schematic diagram of the Drude water model. " $D$ " is drude oscillator particle, " $\mathrm{M}$ " is rigid virtual massless charge point. The bond length is $0.09572 \mathrm{~nm}$ and the $\mathrm{HOH}$ angle is $104.52^{\circ}$

表 1 比较 SPC/E 和 Drude 两种水分子模型所预言的块体物相的分子 偶极矩与介电常数. 压强为 $10^{5} \mathrm{~Pa}$, 温度为 $300 \mathrm{~K}$

Table 1 Comparison of the predictions (using SPC/E and Drude water models) for the bulk phases properties (dipole moments and dielectric constants) of water. At ambient pressure and temperature $\left(10^{5} \mathrm{~Pa}\right.$ and 300 $\mathrm{K})$

\begin{tabular}{cccc}
\hline & $\mathrm{SPC} / \mathrm{E}$ & Drude & Expt. $^{c}$ \\
\hline$\mu_{\text {Gas. }} /($ Debye $)$ & $2.35^{a}$ & $1.85^{b}$ & 1.85 \\
$\mu_{\text {Liq. }} /($ Debye $)$ & $2.35^{a}$ & $2.46^{b}$ & 2.95 \\
$\varepsilon_{0}$ & $71(3)$ & $78(2)$ & 78.4 \\
\hline
\end{tabular}

${ }^{a} \mathrm{SPC} / \mathrm{E}$ model, gas-phase dipole moment $\mu_{\text {Gas }}$ and liquid-phase dipole moment $\mu_{\text {Liq. }}$ taken from reference [20]. ${ }^{b}$ Drude model, gas-phase dipole moment $\mu_{\text {Gas }}$, liquid-phase dipole moment $\mu_{\text {Liq. }}$ taken from reference [21]. ${ }^{c}$ Experimental values, gas-phase dipole moment $\mu_{\text {Gas }}$ from reference [25], dipole moment $\mu_{\text {Liq. }}$ from reference [26], static dielectric constant $\varepsilon_{0}$ from reference [27].

的是, 本工作采用的经典 Drude 模型(基于经典力学) ${ }^{[24]}$ 仍具有一定的缺陷和不足. 例如, 它所给出的原子极化 率是各向同性的, 无法精准描述高阶极化率, 无法完美 再现强场梯度和高阶极矩作用下的原子间色散力. 针对 这些不足, 量子 Drude 模型近年来浮出水面 ${ }^{[28]}$, 但尚未 被广泛应用，计算效率也有待提升。

\section{2 分子动力学模拟方法}

单分子层受限冰和受限水的限域尺寸和受限压强 采用参考文献 [17]和 [18]中的参数设定, 限域尺寸为 $0.65 \mathrm{~nm}$, 限域压强为 $5 \times 10^{8} \mathrm{~Pa}$. 这个受限条件借鉴了 Algara-Siller 等 ${ }^{[16]}$ 实验观测结论, 高限域压强由双层石 墨烯间范德华力致使, $0.65 \mathrm{~nm}$ 是当前实验上可获得单 分子层受限水的最小尺寸. 另外, Algara-Siller 等还证实 过单分子层方冰结构不依赖于受限腔壁的结构(简单的 刚性墙或是柔性石墨烯)、墙-水的相互作用强度以及疏 水性.

与参考文献[17]和[18]中做法一致, 我们将水分子 置于两平行刚性壁内, 刚性腔壁无原子结构 ${ }^{[29,30]}$, 限域 腔壁与受限水分子相互作用采用 10-4-3 势能函数 ${ }^{[31]}$,

$$
\begin{aligned}
\Psi_{\mathrm{wo}}(z)= & 2 \pi \epsilon_{\mathrm{wo}}\left(\frac{4}{5}\right)\left[\frac{2}{5}\left(\frac{\sigma_{\mathrm{wo}}}{z}\right)^{10}-\left(\frac{\sigma_{\mathrm{wo}}}{z}\right)^{4}\right. \\
& \left.-\frac{\sqrt{2}}{3\left(\frac{z}{\sigma_{\mathrm{wo}}}+0.61 \sqrt{2} \frac{z}{\sigma_{\mathrm{wo}}}\right)^{3}}\right]
\end{aligned}
$$

下标中 $\mathrm{w}$ 代表受限墙, $\mathrm{o}$ 代表氧原子. $z$ 表示水分子中氧 原子到腔壁的垂直距离. 对于 $\mathrm{SPC} / \mathrm{E}$ 水分子模型, $\epsilon_{\mathrm{wo}}=0.261856 \mathrm{kcal} / \mathrm{mol}, \sigma_{\mathrm{wo}}=0.326747 \mathrm{~nm}$. 对于 Drude 水分子模型, $\epsilon_{\mathrm{wo}}=0.288018 \mathrm{kcal} / \mathrm{mol}$, $\sigma_{\mathrm{wo}}=0.328211 \mathrm{~nm}$. 表达式 (1) 和势参数是先利用 Lorentz-Berthelot 规则计算出碳原子和水分子的氧原子 之间的相互作用 ${ }^{[32]}$, 再积分单层刚性六元碳环中碳原 子与近邻水分子中氧原子间的碳一氧相互作用获得. 10-4-3 势能函数描述限域腔壁-受限水分子间相互作用, 相比传统的 Lennard-Jones 势函数更准确. 与此同时, 相 比模拟中引入石墨烯，这种简单模型方法能够做到把握 物理本质的同时减少计算量.

分子动力学模拟盒子的构建基于直角坐标系. 盒子 中包含单层水分子以及上下各一个刚性腔壁提供限域. 定义 $z$ 方向垂直于单层水分子和刚性腔壁表面, 水分子 水平分布在 $x y$ 平面方向. 模拟盒子在 $x y$ 方向上应用周 期性边界条件, $z$ 方向上没有应用周期性边界条件. $z$ 方 向盒子的尺寸为 $0.65 \mathrm{~nm}$, 等同于两个刚性腔壁表面间 距. $x$ 和 $y$ 方向盒子尺寸均为 $4.4 \mathrm{~nm}$. 盒子中有 256 个水 分子. 为了精确计算长程静电相互作用, 我们使用 Particle-Particle Particle-Mesh 算法 ${ }^{[33]}$, 并应用了 Yeh 等 ${ }^{\left[{ }^{[34}\right.}$ 提升 Particle-Particle Particle-Mesh 算法精度的技巧, 在 $z$ 方向上引入一个尺度约为 $5 \mathrm{~nm}$ 的真空区域用以去 除模拟盒子与其各个镜像间的偶极-偶极相互作用.

本工作中所有分子动力学模拟均通过美国 Sandia 国家实验室开发的 LAMMPS 软件实现 ${ }^{[35]}$. 模拟过程中, 应用 SHAKE 算法 ${ }^{[36]}$ 来实现 SPC/E 和 Drude 水分子 (Drude 弹簧振荡粒子之外部分)运动的刚性约束条件. 氢氧键键长和键角维持不变. Drude 振荡粒子由单独的 弹性约束运动方程来进行时间积分. 两种体系模拟积分 时间步长取 $1 \mathrm{fs}\left(10^{-15} \mathrm{~s}\right)$. SPC/E 水分子模型体系利用 Nosé-Hoover 热浴恒温法控温, 利用 Anderson 恒压法控 压 ${ }^{[17]}$. Drude 水分子模型体系则相对复杂一些，需要采 用双朗之万控温和控压方法 ${ }^{[37]}$ 才能够避免虚拟的 Drude 振荡粒子与系统真实粒子达到热平衡状态, 而进行非物 理的能量交换导致错误的动力学演化行为. 在具体控温 中, 非 Drude 粒子温度控制在目标温度, 弛豫时间为 0.1 ps; Drude 粒子温度控制在远小于目标温度的低温数值, 弛豫时间为 $0.02 \mathrm{ps}$.

针对两套水分子模型，本工作分别模拟了 4 组不同 的温度的固相 $(100 \mathrm{~K} 、 200 \mathrm{~K} 、 300 \mathrm{~K} 、 400 \mathrm{~K})$ 和液相 $(400$ $\mathrm{K} 、 450 \mathrm{~K} 、 500 \mathrm{~K} 、 550 \mathrm{~K}$ )受限水体系. 调控限域水 $x$ 
和 $y$ 方向上压强维持在 $5 \times 10^{8} \mathrm{~Pa}$. 对每组体系先使用 $\mathrm{NP}_{x y} \mathrm{~T}$ 系综模拟 $10 \mathrm{~ns}$ 使之达到结构、热力学和静电力

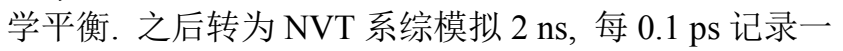
次所有粒子坐标信息用以后续统计分析与计算. 需要说 明的是, 在 Drude 水分子模型体系会观察到众所周知的 (由扩展拉格朗日方法造成)的飞冰(flying-ice-cube)问题, 为了解决这个问题, 需要每 $5 \mathrm{ps}$ 去除一次系统整体平动 与转动动量.

为了和受限水体系比较, 我们利用模拟了 768 个 $\mathrm{SPC} / \mathrm{E}$ 或 Drude 模型水分子组成的液体水和六角相冰结 构. 控温、控压、动力学积分步长和平衡态模拟时长均 与受限水体系保持一致. 因为模拟盒子是三个方向应用 周期性边界条件，仅应用 Particle-Particle Particle- Mesh 算法来处理长程静电相互作用. $x, y, z$ 三个方向盒子尺 寸大约在 $2.7 \mathrm{~nm} 、 3.1 \mathrm{~nm} 、 2.9 \mathrm{~nm}$ 附近(随温度变化).

此外，本工作额外模拟了不同温度下的单层石墨烯 作为受限腔壁的(Drude 模型)单分子层受限水和受限冰, 用以检查受限条件对 Drude 模型水受限水分子极性变化 的影响. 采用两组 720 个碳原子的单层石墨烯(上下两 层相距 $0.65 \mathrm{~nm}$ ) 代替无原子结构的刚性墙. 水分子数目 在模拟中可改变以维持压强恒定. 碳原子在模拟中始终 固定, 仅与水分子中氧原子通过 Lennard-Jones 势函数 相互作用 ${ }^{[32]}$. 长程静电相互作用的处理、模拟温压数值、 平衡态模拟时长均与刚性墙受限水体系一致.

\section{3 受限水介电性质的计算方法}

电介质的介电常数是定义极化矢量和外加电场之 间关系的一个宏观物理量. 当体系具有非均质性或各向 异性时, 介电常数也不再是一个简单量值. 例如本论文 中所研究的准二维受限系统, 介质在 $z$ 方向对称性缺失, 介电常数需要写成张量形式 ${ }^{[11]}$,

$$
\varepsilon=\left(\begin{array}{ccc}
\varepsilon_{x} & 0 & 0 \\
0 & \varepsilon_{y} & 0 \\
0 & 0 & \varepsilon_{z}
\end{array}\right)
$$

其中 $x y$ 和 $z$ 分别表示平行于和垂直于限域腔壁的分量方 向, 极化密度矢量和受限腔体中介质层内电场强度的响 应关系也分为平行和垂直两种分量,

$$
\begin{aligned}
\boldsymbol{P}_{x} & =\frac{\varepsilon_{x}-1}{4 \pi} \boldsymbol{E}_{x} \\
\boldsymbol{P}_{y} & =\frac{\varepsilon_{y}-1}{4 \pi} \boldsymbol{E}_{y} \\
\boldsymbol{P}_{z} & =\frac{1}{4 \pi} \frac{\varepsilon_{z}-1}{\varepsilon_{z}} \boldsymbol{E}_{z}
\end{aligned}
$$

Ballenegger 和 Hansen ${ }^{[11]}$ 利用统计物理方法(配分函数求 平均值)获得了极化密度矢量分量的表达式,

$$
\boldsymbol{P}_{\alpha=x, y, z}=\frac{1}{k_{\mathrm{B}} T} \sum_{\gamma=x, y, z}\left[\left\langle\boldsymbol{m}_{\alpha} \boldsymbol{M}_{\gamma}\right\rangle-\left\langle\boldsymbol{m}_{\alpha}\right\rangle\left\langle\boldsymbol{M}_{\gamma}\right\rangle\right] \boldsymbol{E}_{\gamma}
$$

其中 $\boldsymbol{m}$ 和 $\boldsymbol{M}$ 分别代表电解质的瞬时局域极化密度和偶
极矩总和，尖括号代表时间平均. 将式(5)带入(3)和(4), 即可获得受限偶极分子电介质的介电张量分量的计算 式:

$$
\begin{aligned}
& \varepsilon_{x}=1+\frac{4 \pi}{k_{\mathrm{B}} T}\left[\left\langle\boldsymbol{m}_{x} \boldsymbol{M}_{x}\right\rangle-\left\langle\boldsymbol{m}_{x}\right\rangle\left\langle\boldsymbol{M}_{x}\right\rangle\right] \\
& \varepsilon_{y}=1+\frac{4 \pi}{k_{\mathrm{B}} T}\left[\left\langle\boldsymbol{m}_{y} \boldsymbol{M}_{y}\right\rangle-\left\langle\boldsymbol{m}_{y}\right\rangle\left\langle\boldsymbol{M}_{y}\right\rangle\right] \\
& \varepsilon_{z}^{-1}=1-\frac{4 \pi}{k_{\mathrm{B}} T}\left[\left\langle\boldsymbol{m}_{z} \boldsymbol{M}_{z}\right\rangle-\left\langle\boldsymbol{m}_{z}\right\rangle\left\langle\boldsymbol{M}_{z}\right\rangle\right]
\end{aligned}
$$

从分子动力学模拟中每 $0.1 \mathrm{ps}$ 记录的所有粒子的坐标信 息, 可以获得式(6)和(7)中极化密度分量 $\boldsymbol{m}_{x}, \boldsymbol{m}_{y}$ 和 $\boldsymbol{m}_{z}$ 以及总偶极矩分量 $\boldsymbol{M}_{y}, \boldsymbol{M}_{y}$ 和 $\boldsymbol{M}_{z}$ 的瞬时数值信息, 从而 统计计算出单分子层受限水的介电张量 $\varepsilon_{x}, \varepsilon_{y}$ 和 $\varepsilon_{z}$.

介电常数之外, 我们还计算了 $x y$ 平面内径向偶极偶极关联函数 ${ }^{[13]}$ 用以分析受限水分子的偶极方向结构 性质,

$$
F(r)=\frac{1}{N}\left\langle\sum_{i=1}^{N} \frac{1}{n_{i}(r)} \sum_{j=1}^{n_{i}(r)} \widehat{\boldsymbol{m}}_{i} \cdot \widehat{\boldsymbol{m}}_{j}\right\rangle
$$

表达式中 $n_{i}(r)=\left\langle\delta\left(r_{i}-r\right)\right\rangle$ 表示 $x y$ 平面内距离水分子 $i$ 为 $r$ 到 $(r+\mathrm{d} r)$ 范围内的分子数目, $\widehat{\boldsymbol{m}}_{i}$ 和 $\widehat{\boldsymbol{m}}_{j}$ 代表第 $i$ 个和 $j$ 个水分子的约化偶极矩(除模长), $N$ 是水分子总数目.

本工作研究的最后一个介电性质是受限水的德拜 (偶极)弛豫时间 $\tau_{\alpha=x, y, z}$, 即系统 $x y z$ 方向上总偶极矩时 间(自)关联函数 $\Phi_{\alpha}(t)$ 的弛豫时间. 关联函数 $\Phi_{\alpha}(t)$ 的计 算表达式如下,

$$
\Phi_{\alpha}(t)=\frac{\left\langle M_{\alpha}(0) \cdot M_{\alpha}(t)\right\rangle}{\left\langle M_{\alpha}^{2}\right\rangle}
$$

使用指数函数 $\exp \left(-t / \tau_{\alpha}\right)$ 拟合 $\Phi_{\alpha}(t)$, 可以提取出德拜 弛豫时间 $\tau_{\alpha}$. 需要说明的是, 受限水体系 $\Phi_{z}(t)$ 呈周期性 正负交替振荡结构，具体拟合过程中，选取震荡结构中 所有峰值进行拟合.

\section{3 结果与讨论}

\section{1 模拟坐标快照}

图 2 中记录了 NVT 系综模拟中某一个瞬时时刻单 分子层受限冰(a), (b) 和受限水(c), (d) 的 $x y$ 平面坐标快照. $T=400 \mathrm{~K}$. 图 2(a)和(c)为 SPC/E 水分子模型结果, 图 2(b) 和(d)为 Drude 极化水分子模型结果. 图 2 中用红色代表 受限冰，蓝色代表受限液相水，下文图中同样使用红蓝 颜色区分受限冰和水. 恒定偶极矩与极化水分子模型模 拟的受限冰均呈现与 Algara-Siller等工作中(分子模拟部 分结果)非常一致的结构, 即长程有序的平面晶格结构, 氢氧键的方向集中在 $x y$ 平面内. 液相限域水中, 两种水 模型体系均呈现长程无序性结构, 平动与转动型扩散远 大于受限冰. 在 $\mathrm{SPC} / \mathrm{E}$ 水模型描述的受限水中, 观察到 
皮秒量级短时间尺度，纳米空间尺度局域有序的才簇结 构(10 到 20 个水分子)集体形式地参与密度涨落与质量 输运. 在 Drude 极化水模型体系中, 局域瞬态有序的团 族结构仍可被观察到, 但似乎尺寸要小一些、生存时间 更短一些.

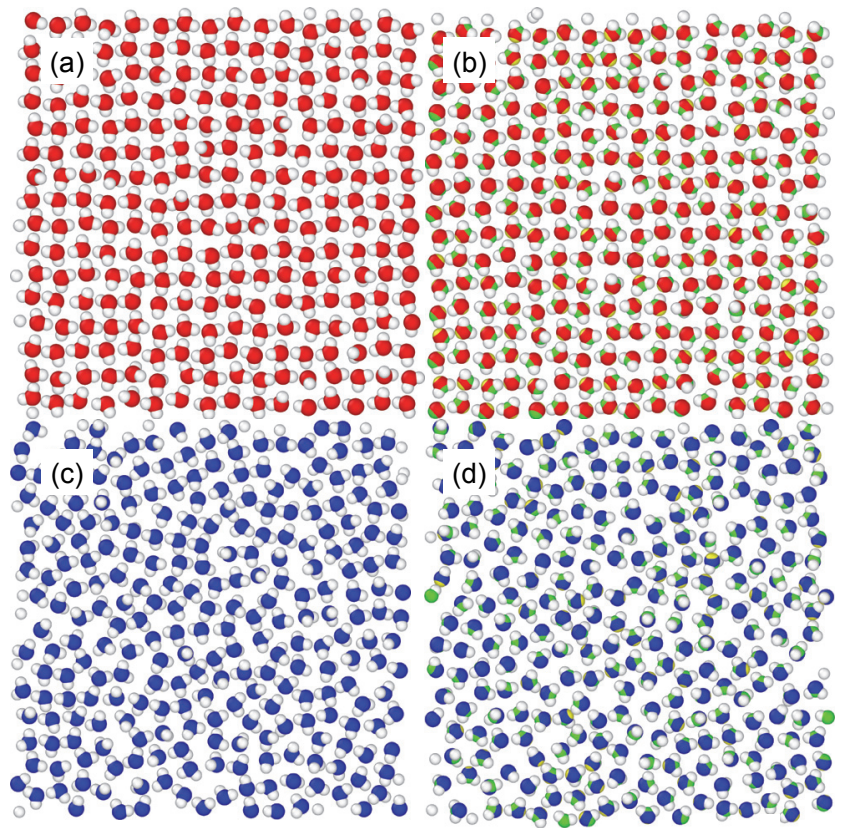

图 2 单分子层受限冰和受限水的平衡态分子动力学模拟坐标快照. (a), (c)分别是 SPC/E 水分子模型模拟的受限冰和受限水. (b), (d) 分别 是 Drude 极化水分子模型模拟的受限冰和受限水. 温度 $400 \mathrm{~K}$, 压强 $5 \times 10^{8} \mathrm{~Pa}$. 白色球为氢原子, 红色球为受限固相冰中水分子内氧原子, 蓝色球为受限液相水分子中氧原子. 绿色球为 Drude 水分子模型中 $M$ 点, 黄色球为 Drude 水分子模型中的 Drude 振荡粒子.

Figure 2 Snapshot of monolayer square ice and water confined within two from the equilibrium molecular dynamics simulation. Panel (a) and (c): simulation results using SPC/E model potential, panel (b) and (d): simulation results using SWM4-NDP polarizable model. Confined pressure is $5 \times 10^{8} \mathrm{~Pa}$ and simulation temperature is set as $400 \mathrm{~K}$. Hydrogen atoms are represented with white spheres, oxygen atoms are depicted as red (crystalline phase) and blue (liquid phase), respectively. Masslees site "M" and Drude oscillator particle in SWM4-NDP model are represented with the green and yellow spheres.

$\mathrm{SPC} / \mathrm{E}$ 水分子模型的偶极矩为恒定数值 2.35 Debye, 它不随物相、温度、受限条件发生任何变化. 而 Drude 极化水模型的显著优势即能够展现物相对分子极性的 强烈影响, 前言中介绍的其它两类极化水模型也都能够 很好地描述液、汽两相中水分子不同的偶极矩数值. 汽 相水分子间距远大于液相水中分子间距，因此分子极性 的明显变化是容易理解的. 图 3(a)中, 我们计算了 Drude 水分子模型模拟常压下块体水和六角晶格块体冰 相的分子极性统计结果. 其中插图是分子偶极矩平均值 随温度的变化结果. 图 3(a)中的结果展现出水分子偶极 矩的分布呈正态分布, 温度越高分布中值逐渐左移变小, 分布范围变宽. 可以理解为水分子瞬态极性数值的涨落 和其在凝聚态物相中平动和转动自由度的扩散能力关 联. 从 $50 \mathrm{~K}$ 到 $400 \mathrm{~K}$ 共 $350 \mathrm{~K}$ 的温度变化范围内, 偶极
矩的平均值从 2.62 Debye 减小到了 2.34 Debye, 减小幅 度近 0.3 Debye. 固相和液相区域的温度梯度变化斜率 不等, 液相温度变化梯度更大. 在固-液相变温度处, 固-液两相水分子的偶极矩平均值相等, 并未像密度和 势能等热力学量呈现跳变(块体水-冰的固-液结构相变 是一级相变), 而是展现出连续变化形式.

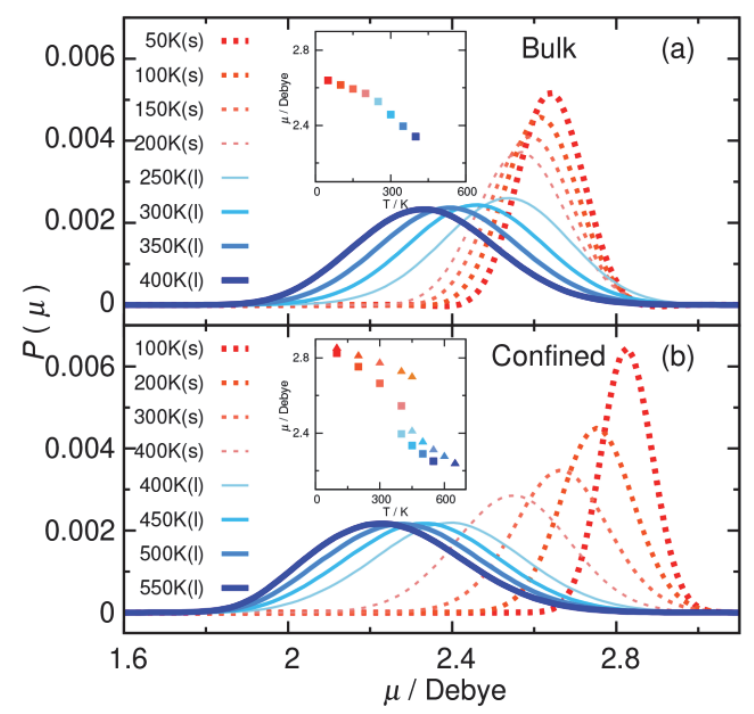

图 3 Drude 极化模型水分子偶极矩随温度和物相的变化统计结果. (a) 不同温度下, 常压下块体水(蓝色)和六角晶格块体冰(红色)的水分子 瞬时偶极矩概率密度分布函数. (b) $5 \times 10^{8} \mathrm{~Pa}$ 受限压强, 不同温度下, 单分子层受限水(蓝色)和受限冰 (红色)中水分子瞬时偶极矩概率密度 分布函数. (a)和(b)中插图为水分子平均偶极矩和温度的变化关系, (b) 插图中三角形为石墨烯受限腔壁模拟结果

Figure 3 The calculated polarity variation of the water molecules, described by SWM4-NDP polarizable model, with changing the temperature and (confined) structural phases. Panel (a): measured probability distributions of the instantaneous water molecular dipole moments, blue lines represent bulk water phase at ambient pressure while red lines represent bulk hexagonal ices. Panel (b): under $5 \times 10^{8} \mathrm{~Pa}$ confinement pressure, blue lines represent probability results of confined monolayer liquid water phases, red lines represent results of confined monolayer ice phases. Inset figures in (a) and (b) show average dipole moments as the function of the temperature. Triangle symbols in the inset figure in (b) represent simulation results with graphene-confinement.

\section{2 单分子层受限水分子极性的奇异变化}

然而，在单分子层受限体系中的计算结果让我们非 常吃惊. 图 3(b)中两处结果与块体体系明显的差异: (1) 相同温度变化范围 $(100 \mathrm{~K}$ 到 $450 \mathrm{~K}, 350 \mathrm{~K}$ 温区)的水分 子偶极矩平均值从 2.84 Debye 降到 2.34 Debye, 减幅 0.5 Debye 明显大于块体水体系, 说明温度对受限水体系的 极性变化影响更大. (2)更意想不到的是，在 $400 \mathrm{~K}$ 受限 冰和受限水两相共存温度下, 水分子偶极矩平均值出现 跳变，与块体体系的过渡方式完全不同.

此区别意味着单分子层受限水-冰结构相变过程中, 固-液界面处可能伴随着偶极矩分布的不均匀性，偶极 矩数值变化与结构相变可能是协作发生的. 为了验证这 一猜测, 利用本文作者开发的低维受限水-冰固-液平衡 
态分子动力学模拟技术 ${ }^{[17]}$, 参考文献[17]中的分子动力 学模拟细节参数设定并结合本工作所使用的 Drude 极化 水分子模型参数和腔壁-水分子间相互作用形式, 我们 实现了 Drude 极化水分子模型描述的单分子层受限冰水(固-液)两相平衡态模拟. 如图 4(a)所示, 色谱颜色变 化标注的水分子/氧原子的结构序参量(计算方法详见参 考文献[17]). 红色代表高序参量数值的固相, 蓝色代表 低序参量数值的液相. 结构序参量中色彩变化可直观表 征出固-液界面的位置. 在 $5 \mathrm{~ns}$ 的平衡态模拟中, 固-液 界面始终稳定存在, 说明当前单分子层限域冰一水体系 经历了一级(固-液)相变. 通过统计分析 Drude 水分子偶 极矩沿着界面法向方向上的分布函数(参照参考文献 [17] 中列热力学参量沿 $x$ 轴分布函数的计算方法), 见图 4(b), 我们发现受限冰和受限水偶极矩数值处于不同的平台 值, 偶极矩数值变化与结构相变是协作发生的. 由此可 以推断单分子层受限冰和水的分子偶极矩的跳变, 相较 $\mathrm{SPC} / \mathrm{E}$ 等恒定偶极矩水分子模型体系, 将会导致受限冰、

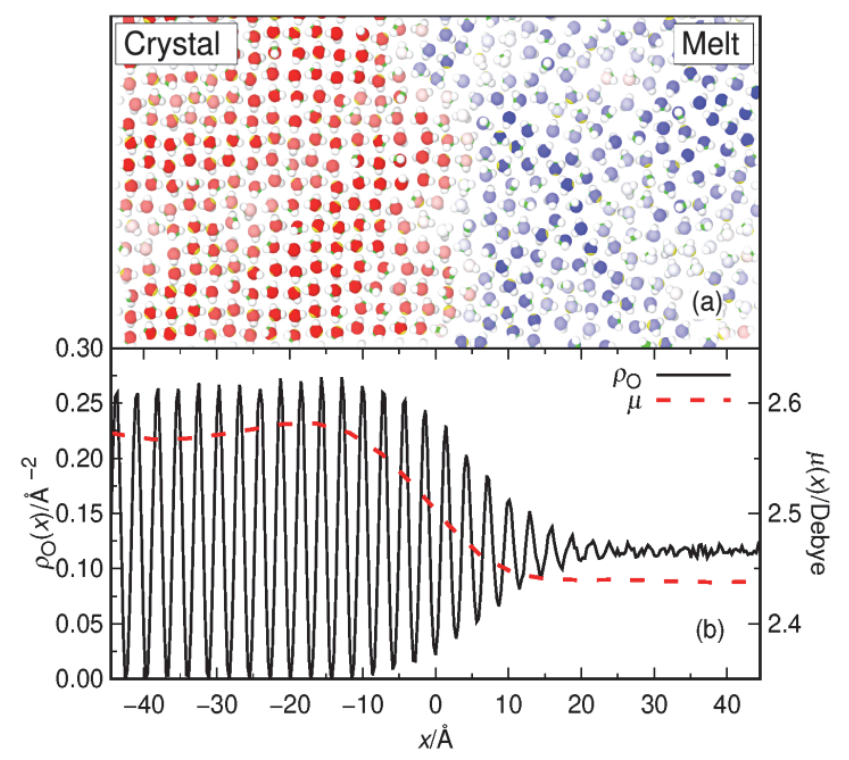

图 4 (a) Drude 极化水分子模型单分子层限域冰-水相平衡分子动力 学模拟分子坐标快照. 压强 $5 \times 10^{8} \mathrm{~Pa}$ 、温度 $400 \mathrm{~K}$. 氧原子颜色变化 遵循结构序参量数值, 红色代表固相, 蓝色代表液相. (b)单分子层限 域冰-水相平衡平面内细粒氧原子密度分布函数(实线)和粗粒偶极矩 分布函数(虚线). $x$ 轴 0 点对应固液界面位置. 结构序参量和粗细粒度 分布函数计算方法参见文献[17]

Figure 4 (a) SWM4-NDP polarizable model, snapshot of the monolayer confined ice-water equilibrium interface obtained from a molecular dynamic simulation. Lateral pressure $5 \times 10^{8} \mathrm{~Pa}$, under a coexistence temperature of $400 \mathrm{~K}$. Oxygen atoms in (a) is colored coded based on the value of the structural order parameter, red for crystal and blue for melt. (b) In-plane fine-grained area-density of oxygen atoms profile (solid line) and coarse-scaled profiles for dipole moment of SWM4-NDP water (dashed line) for the monolayer confined ice-water coexistence. The zero point of $x$ corresponding to the time averaged position of the crystal-melt coexistence interface. The calculation methods for the structural order parameter as well as the fine/coarse-scaled profiles can be found in the Ref. [17]
水两相的化学势差别增大, 两相形成界面自由能要更 高 $^{[38]}$, 从而影响到受限冰形核形貌和晶体生长速度.

需要说明的是, 国内外不乏关于受限水(或冰)体系 水分子重要物理化学性质异于块体水(或冰)的报道, 例 如, Luo 和 Zeng 等 ${ }^{[39]}$ 发现的铁电极化受限冰, Wang 等 ${ }^{[40]}$ 预言的受限水分子 $\mathrm{O}-\mathrm{H}$ 键振动频率在受限腔壁离域 $\pi$ 键作用下发生红移效应等. 本工作中关于水分子极性随 温度和固-液结构变化方式在受限条件下显著区别于块 体体系的理论预言, 和 $\mathrm{Luo}^{[39]}$ 和 $\mathrm{Wang}^{[40]}$ 等的研究工作 一样，一定程度上丰富了对受限水体系奇特结构物性的 认识. 显而易见, Drude 极化水分子模型描述的受限维 度对水分子极性的强烈影响效应，是恒定偶极矩的 $\mathrm{SPC} / \mathrm{E}$ 模型无法企及的. 为了进一步证实这个发现不依 赖于受限条件的模拟细节，我们额外模拟了单层石墨烯 作为受限腔壁的(Drude 模型)单分子层受限水和受限冰, 观察到 $450 \mathrm{~K}$ 温度下两相水分子偶极矩平均值更显著的 跳变, 见图 3(b)插图中三角形数据点. 最后, 我们需要 指出的是, 经典的 Drude 极化水分子是针对液体结构和 动力学进行优化设计的, 并不能保证其具备精准描述单 分子偶极矩的能力. 偶极矩的变化本质上来源于电子结 构的变化, 要严格研究这一单分子层受限冰-水相变带 来的偶极矩跳变的问题, 需要考虑量子效应的影响.

\section{3 单分子层受限冰与受限水的结构}

图 5 比较计算了单分子层受限冰氧原子结构因子 $(b \sim e, g \sim i)$, 左右两列 $(S P C / E$ 和 Drude 水分子模型)结 构因子结果具有非常相似的强度分布结构, 高波数峰随 温度升高而退化的行为(温度接近熔点, 受限冰长程有

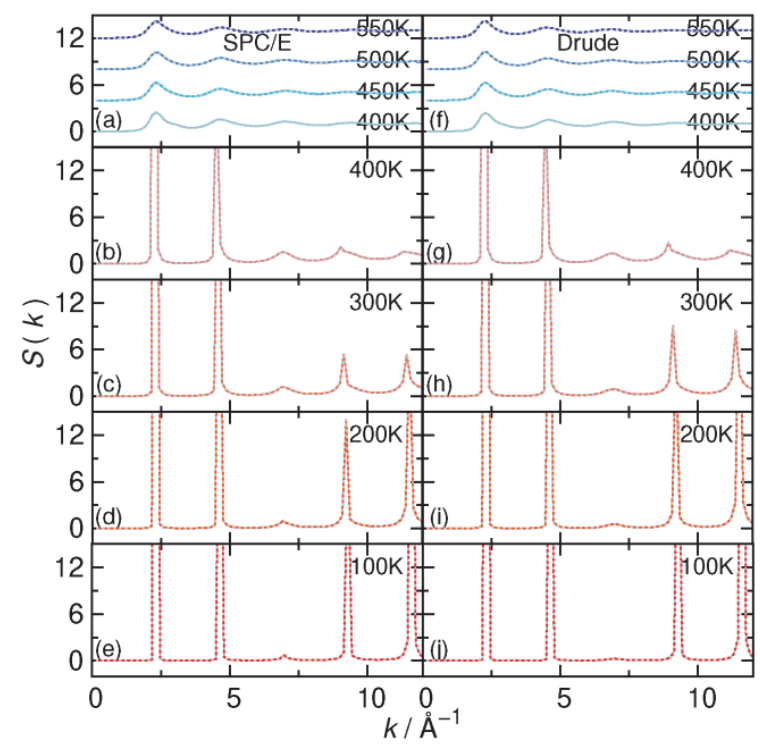

图 5 单分子层受限水 $(a, f)$ 和受限冰 $(b \sim e, g \sim j)$ 氧一氧原子结构因子. 左侧 SPC/E 水分子模型, 右侧 Drude 极化水分子模型

Figure 5 The calculated oxygen-oxygen atomic structural factors for the monolayer confined water $(a, f)$ and ices $(b \sim e, g \sim j)$. Left column: results of SPC/E model systems, right column: results of polarizable SWM4-NDP polarizable model systems 
序逐渐消失)也非常接近. 值得注意的是, 结构因子第 三个峰位 $\left(k \approx 7 \AA^{-1}\right)$ 的极弱信号意味着受限冰的晶格 偏离了正方晶格, 而呈现锯齿状排列的棱形结构. 关于 使用恒定偶极矩水分子模型的经典分子动力学模拟能 否预测实验观测的严格方形晶格, 已有过大量报道讨论, 至今还存在争议 ${ }^{[41]}$. $\mathrm{Wu}$ 和 $\mathrm{Geim}$ 等 ${ }^{[42]}$ 认为亚纳秒时间尺 度的模拟统计平均不足以预言实验的成像. Michaelides 等 ${ }^{[43]}$ 开展第一性原理计算引领诸多研究讨论严格方形 单分子层受限冰结构相的理论可能. 从图 5 计算模拟结 果来看, 具有极化能力的 Drude 水分子模型也无法获得 所预言的瞬时严格方形冰结构物相. 我们认为核量子效 应导致的氢键网络畸变在这个方冰结构模拟问题中可 能扮演关键角色, 需要更高精度级别的水模型, 例如量 子 Drude 模型 ${ }^{[24]}$ 等. 作为参考, 图 5(a, f) 列出各温度下 受限水氧原子结构因子.

图 6 比较了 $\mathrm{SPC} / \mathrm{E}$ 和 Drude 水分子模型模拟单分子 层受限水 $x y$ 平面内径向偶极 - 偶极关联函数 $F(r)$. 两种 水分子模型的 $F(r)$ 结果在相同的温度下非常相似, 随温 度变化的形状调节形式也非常接近. 在特定温度下, 例 如 $400 \mathrm{~K}$, 虚线描述的 $F(r)$ 呈现非均匀的空间关联结构 模式, 在 $1.2 \mathrm{~nm}$ 范围内能看到 4 个明显的关联峰谷结构. 随温度升高关联强度有微小的减弱, Drude 模型体系相 比 SPC/E 模型体系关联性要更弱一些. 观察 $F(r)$ 关联函 数的分量, 我们发现强烈的各向异性. $F(r)$ 的 $z$ 分量结 果几乎都为 0 相关, 只有 $0.3 \mathrm{~nm}$ 距离附近有一个极其微 弱的负相关峰. $F(r)$ 的主要结构特征都来自于其 $x y$ 分 量. 这个强烈的各向异性反映出受限水的单分子层平面 排列方式，在 $z$ 方向上自由度受到极大的限制.

通过图 5 和图 6 的比较分析, 我们可以获得一个结 论, 即极化水分子模型在描述受限冰和受限水的物相的 结构性质时, 和 $\mathrm{SPC} / \mathrm{E}$ 等恒定偶极矩水分子模型效果相 当, 和水分子极性的涨落变化无明显关系. 因此我们建 议如果开展仅限于受限水物相结构性质的模拟研究的 话, 选用传统恒定偶极矩水分子模型进行模拟足以胜任, 且更节省计算资源.

\section{4 介电张量计算结果}

我们用公式(6)和(7)计算了单分子层受限冰和受限 水的介电张量分量, $5 \times 10^{8} \mathrm{~Pa}$ 受限压强、不同温度下 $\mathrm{SPC} / \mathrm{E}$ 水分子模型和 Drude 极化水分子模型体系的比较 结果都在表 2 中详细列出. 表 2 前 4 行数据比较了受限 冰的介电张量分量. 由于处于固相的水分子几乎没有平 动和转动的能力, $100 \mathrm{~K}$ 到 $400 \mathrm{~K}$ 介电张量分量数值都 非常接近 1 , 但最大数值只有 1.7 , 低于质子有序块体冰 的介电常数数值 $(3 \sim 4)^{[44]}$. 低温下 $\varepsilon_{z}$ 比 $\varepsilon_{x}$ 或 $\varepsilon_{y}$ 稍大, 这 个差别随着温度的升高而变小. 这说明在当前模拟的受 限与热力学条件下, 受限冰自身的晶体结构对介电常数 的限制比受限腔体对介电常数的限制更大一些. 仅从受

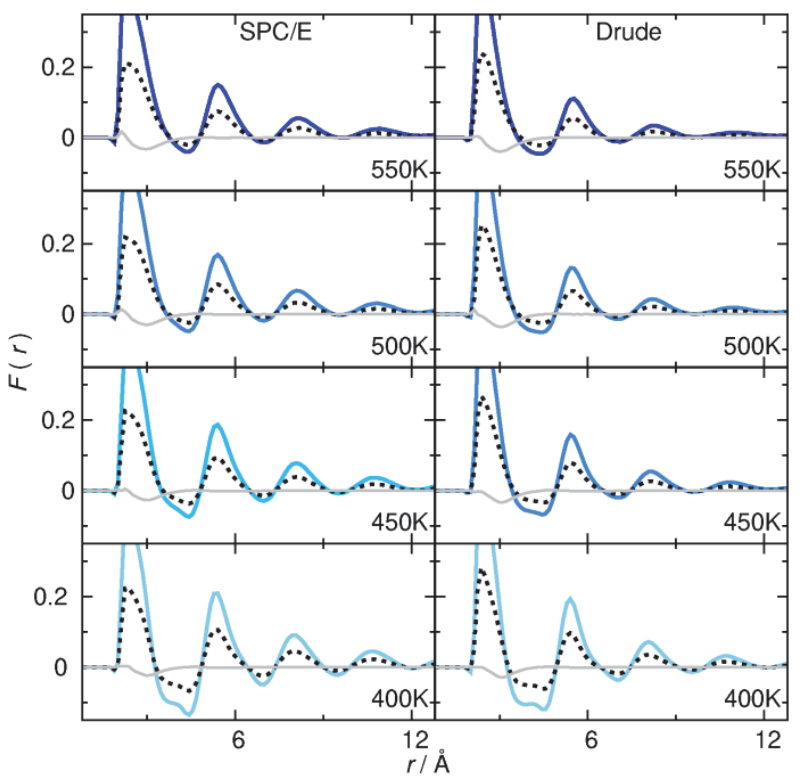

图 6 单分子层受限水的径向偶极-偶极关联函数. 左侧 $\mathrm{SPC} / \mathrm{E}$ 水分 子模型，右侧 Drude 极化水分子模型. 虚线、浅灰实线和蓝色实线分 别代表 $x y$ 方向、 $z$ 方向分量和所有方向平均结果

Figure 6 Radial dipole-dipole correlation function (blue solid lines) and its $x y$ (dashed lines) and $z$ (grey lines) components of monolayer confined water. Left column: results of SPC/E model systems, right column: results of SWM4-NDP polarizable model systems

表 24 个温度下单分子层受限冰(用 $\mathrm{s}$ 表示)和受限水(用 1 表示) $x y z$ 三个方向的介电张量分量的计算结果. 第 $3 \sim 5$ 列是 $\mathrm{SPC} / \mathrm{E}$ 水分子模型模拟体 系的结果, 第 6 8 列是 Drude 极化水分子模型模拟体系的结果. 括号内数字代表 95\%置信度的统计误差

Table 2 Calculated dielectric tensor data of the monolayer confined ice (labeled with "s" for the solid phase) and water (labeled with "l" for the liquid phase), for the four temperatures and $x y z$ components. The 3rd to 5th column present results of the SPC/E model systems, the 6th to 8th column present results of the SWM4-NDP polarizable model systems. Numbers in parentheses are $95 \%$ confidence errors for the last digits shown

\begin{tabular}{|c|c|c|c|c|c|c|c|}
\hline \multirow{2}{*}{$T / \mathrm{K}$} & \multirow{2}{*}{ Phase } & \multicolumn{3}{|c|}{$\mathrm{SPC} / \mathrm{E}$} & \multicolumn{3}{|c|}{ Drude } \\
\hline & & $\varepsilon_{x}$ & $\varepsilon_{y}$ & $\varepsilon_{z}$ & $\varepsilon_{x}$ & $\varepsilon_{y}$ & $\varepsilon_{z}$ \\
\hline 100 & $\mathrm{~s}$ & $1.140(1)$ & $1.143(2)$ & $1.47(2)$ & $1.156(2)$ & $1.156(3)$ & $1.53(1)$ \\
\hline 200 & $\mathrm{~s}$ & $1.177(2)$ & $1.172(3)$ & $1.50(1)$ & $1.167(2)$ & $1.166(4)$ & $1.49(2)$ \\
\hline 300 & $\mathrm{~s}$ & $1.232(5)$ & $1.234(3)$ & $1.60(2)$ & $1.255(7)$ & $1.257(4)$ & $1.54(1)$ \\
\hline 400 & $\mathrm{~s}$ & $1.386(5)$ & $1.399(12)$ & $1.70(1)$ & $1.52(2)$ & $1.54(3)$ & $1.62(1)$ \\
\hline 400 & 1 & $66(4)$ & $63(4)$ & $2.24(4)$ & $54(1)$ & $54(2)$ & $1.95(3)$ \\
\hline 450 & 1 & $59(3)$ & $58(6)$ & $2.17(6)$ & $47(1)$ & $47(1)$ & $1.90(2)$ \\
\hline 500 & 1 & $50(1)$ & $49(2)$ & $2.15(3)$ & $38(1)$ & $38(1)$ & $1.86(2)$ \\
\hline 550 & 1 & $40(1)$ & $41(1)$ & $2.15(5)$ & $29(1)$ & $29(1)$ & $1.85(2)$ \\
\hline
\end{tabular}


限冰的静态介电性质结果完全看不出极化水分子模型 和恒定偶极矩水分子模型之间的差异.

表 2 中后 4 行数据是单分子层受限水介电张量分量 的计算结果. 受限液相水的 $\varepsilon_{x}$ 和 $\varepsilon_{y}$ 分量数据的数量级明 显高于其 $\varepsilon_{z}$ 分量以及受限冰的三个分量, 和表 1 中块体 水的介电常数数量级相同. 两种水分子模型在数值预言 上体现出了更明显差别, Drude 水分子模型预言的 $\varepsilon_{x}$ 和 $\varepsilon_{y}$ 更小一些, 但是有悖于其在块体水介电常数更大数值 的预言. 因为 Geim 的实验并未测量受限水的 $\varepsilon_{x}$ 和 $\varepsilon_{y}$ 分 量数值, 我们不能判断 Drude 模型预言的 $\varepsilon_{x}$ 和 $\varepsilon_{y}$ 是否更 加接近实验数据. 另外, 我们获得的受限水 $\varepsilon_{x}$ 和 $\varepsilon_{y}$ 数值 均小于块体水的介电常数, 并且随温度升高有显著减小, 这与 Galli 等 ${ }^{[13]}$ 受限水分子模拟结果不符(她们报道是受 限尺寸减小 $\varepsilon_{x}$ 和 $\varepsilon_{y}$ 数值增大). Galli 等 ${ }^{[13]}$ 认为受限水密 度对其极化涨落 (即 $\varepsilon_{x}$ 和 $\varepsilon_{y}$ 数值) 行为影响强烈. 我们认 同这一观点, 并认为受限水分子在 $x y$ 平面内的转动并 未受到受限腔体的限制, $\varepsilon_{x}$ 和 $\varepsilon_{y}$ 的数值和随温度的变化 方向问题仍属于块体液体介电性质范畴, 仅需要研究清 楚受限水 $x y$ 平面密度、压强分量和极化强度涨落之间 的物理关联即可.

两种水分子模型预言的单分子层受限液相水 $\varepsilon_{z}$ 数 值差别仅有 0.3 左右, 远小于其在受限液相水 $\varepsilon_{x}$ 和 $\varepsilon_{y}$ 分 量上的预测数值的差别. $\varepsilon_{z}$ 分量数值也远小于块体液体 介电常数和 $\varepsilon_{x}$ 和 $\varepsilon_{y}$ 分量数值, 介电张量强烈各向异性的 主要原因是水分子转动带来的偶极涨落被受限腔壁强 烈限制，水分子偶极矩方向和氢氧成键大都集中在 $x y$ 平面内. 这与之前实验测量 ${ }^{[15]}$ 以及计算模拟 ${ }^{[13]}$ 的研究 发现一致, 介电张量的 $z$ 方向分量强烈依赖受限尺寸. 表格 2 中受限水 $\varepsilon_{z}$ 分量数值随温度有微弱的变化, 通过 多项式外延分析, 我们得到室温下 $\varepsilon_{z}$ 分量数值(Drude 模 型 $\varepsilon_{z}=2.1, \mathrm{SPC} / \mathrm{E}$ 模型 $\left.\varepsilon_{z}=2.5\right)$. Drude 模型的 $\varepsilon_{z}$ 结果和 Geim 等的实验结果(受限尺寸小于 $2 \mathrm{~nm}, \varepsilon_{z}$ 数值为 2.1 \pm 0.2 ) 完全相同. Geim 等认为 ${ }^{[15]}$ 在 $2 \mathrm{~nm}$ 受限尺寸以内, 水分子转动及氢键网络 ${ }^{[45]}$ 对介电张量的贡献完全被抑 制, 有限的介电常数来自独立于受限条件的电子与少量 原子偶极的贡献. 本研究实施的经典分子动力学模拟无 法描述电子云分布和原子偶极, 因此我们认为 Drude 水 分子模型描述单分子层受限水结果与 Geim 实验测量出 结果数值上的相符并不能完全澄清 $\varepsilon_{z}=2.1$ 中所有贡献成 分. 未来有必要采用能够精确描述电子波函数的分子模 拟技术继续探索.

就可极化水分子模型对于恒定偶极矩水分子模拟 的优势而言, 通过上述介电性质的计算结果, 我们获得 一个重要认识, 模型改进(偶极矩变化能力的引入)对于 介电常数描述的优势在分子偶极方向转动未被限制的 情况下可以展现, 当水分子的偶极转动涨落在固相晶格 和受限方向被大幅限制时, 极化模型描述静态介电性质 的优势也被大幅削减. 此外, $400 \mathrm{~K}$ 单分子层受限水固-
液两相介电张量 $\varepsilon_{z}$ 分量数值差别虽然很小 $(0.33)$, 但仍 在误差精度内可分辨，这个差别应会随着受限尺寸的增 加而变大. 这意味着受限冰固一液相变的精准调控有希 望带来介电常数的定量调节, 受限冰相变介电器件存在 理论可行性.

\section{5 受限水的德拜弛豫时间}

最后, 我们计算了偶极矩时间关联函数 $\Phi_{\alpha}(t)$ 及德 拜弛豫时间 $\tau_{\alpha}$ ( $\alpha$ 代表 $x y z$ 中任意一个分量). 表 3 中详细 列出了 $5 \times 10^{8} \mathrm{~Pa}$ 受限压强、不同温度下 $\mathrm{SPC} / \mathrm{E}$ 水分子 模型和 Drude 极化水分子模型体系的 $\tau_{\alpha}$ 计算结果. 我们 发现受限液相水的德拜弛豫时间展现出极强的各向异 性, $x y$ 分量数据和块体水的德拜弛豫时间处于相同的数 量级(实验测量值 $8.2 \pm 0.4 \mathrm{ps}^{[46]}$ ), 但比 $z$ 分量数据高出 两个数量级. 这个各向异性形式类似于上文中受限水介 电张量 $\left(\varepsilon_{x} 、 \varepsilon_{y}\right)$ 和 $\varepsilon_{z}$ 分量的各向异性. 随温度升高各分量 的弛豫时间也相应变小. 我们认为德拜弛豫时间 $\tau_{x y}$ 分 量的变化机理与 $\varepsilon_{x y}$ 相似, 也属于块体液体的介电性质 范畴内的问题, 主要由 $x y$ 平面内水分子的数密度变化 引起而与受限条件无关. 而单分子层受限水 $\tau_{z}$ 分量则不 同于 $\tau_{x}$ 和 $\tau_{y}$ 分量, 其比块体水快两个数量级的电极化弛 豫能力是因为其所处的受限状态, 相较块体水体系, 相 同强度的外电场驱动下, 强受限条件下的水在 $z$ 方向的 整体极化的时间关联大大减小.

表 34 个温度下单分子层受限水(用 1 表示) $x y z$ 三个方向的德拜驰豫 时间计算结果. 第 3 5 列是 $\mathrm{SPC} / \mathrm{E}$ 水分子模型模拟体系的结果, 第 6 8 列是 Drude 极化水分子模型模拟体系的结果. 括号内数字代表 95\%置信度的统计误差

Table 3 Calculated Debye relaxation time of the monolayer confined water (labeled with "l" for the liquid phase) for the four temperatures and $x y z$ components. The 3 rd to 5 th column present results of the SPC/E model systems, the 6th to 8th column present results of the SWM4-NDP polarizable model systems. Numbers in parentheses are $95 \%$ confidence errors for the last digits shown

\begin{tabular}{ccccccccc}
\hline \multirow{2}{*}{$T / \mathrm{K}$} & Phase & \multicolumn{3}{c}{$\mathrm{SPC} / \mathrm{E}$} & & \multicolumn{3}{c}{ Drude } \\
\cline { 8 - 10 } \cline { 7 - 9 } & & $\tau_{x} / \mathrm{ps}$ & $\tau_{y} / \mathrm{ps}$ & $\tau_{z} / \mathrm{ps}$ & & $\tau_{x} / \mathrm{ps}$ & $\tau_{y} / \mathrm{ps}$ & $\tau_{z} / \mathrm{ps}$ \\
\hline 400 & 1 & $10.7(9)$ & $9.7(9)$ & $0.154(3)$ & & $6.4(3)$ & $6.7(3)$ & $0.055(2)$ \\
450 & 1 & $5.6(2)$ & $5.5(5)$ & $0.126(2)$ & & $3.4(3)$ & $3.4(2)$ & $0.042(1)$ \\
500 & 1 & $3.6(2)$ & $3.5(2)$ & $0.112(2)$ & & $2.1(2)$ & $2.1(3)$ & $0.031(4)$ \\
550 & 1 & $2.4(1)$ & $2.5(2)$ & $0.096(4)$ & & $1.3(1)$ & $1.4(2)$ & $0.029(3)$ \\
\hline
\end{tabular}

比较两种水分子模型在描述单分子层受限水动态 介电响应性质上的表现, 我们发现 SPC/E 水分子模型预 言的 $\tau_{x}$ 和 $\tau_{y}$ 数值比 Drude 极化水分子模型预言的数值大 一些，差别形式类似于受限液相水介电张量 $x y$ 分量结 果. 两种水分子模型在 $\tau_{z}$ 分量上预言的结果差别高达三 倍, 引起我们的注意, 为了探究其中缘由, 在图 7 中仔 细比较了两种水分子模型体系 $x y$ 和 $z$ 分量偶极矩时间关 联函数 $\Phi_{x}(t), \Phi_{y}(t)$ 和 $\Phi_{z}(t)$ 的计算结果. 我们发现了两 种水分子模型的 $\Phi_{z}(t)$ 时间演化结构差别巨大. SPC/E 模 型体系的 $\Phi_{z}(t)$ 衰减呈周期性正负交替振荡结构(图 7c), 而块体水和受限水的 $\Phi_{x}(t)$ 和 $\Phi_{y}(t)$ 均为单调的指数衰减 
(如图 7a 和 $7 \mathrm{~b}$ 所示). Drude 模型体系的 $\Phi_{z}(t)$ 时间衰减中 振荡的幅度则明显弱的多, 并未出现强烈的符号变换 (图 7d), 振荡周期更长, 关联函数收玫前出现的周期数 更少. 两种水分子模型在单分子层受限水模拟的 $\Phi_{z}(t)$ 结果中均展现振荡衰减结构, 让我们更倾向真实体系中 也会有类似的行为. 其中原因猜测是单分子层受限水中 的极化密度与水分子数密度的局域非均匀性导致(例如 前文中模拟快照观察到的瞬态局域有序团簇结构), 偶 极极化在 $z$ 方向上的涨落受到局域非均匀性的涨落与涌 现影响. SPC/E 水分子模型所有化学键都为刚性, 极化 涨落直接通过水分子的转动甚至是团簇的整体翻转实 现, 而 Drude 极化水分子模型中的振荡粒子起到了类似 于阻尼减震器的效果, 让受限水偶极极化涨落的弛豫过 程更平稳, 更快地完成. 进一步猜测极化水分子模型的 动态偶极矩变化能力, 可能会在受限水的动态介电性质 的描述中具有优势, 但是这一猜测需要实验技术的进一 步升级(只能测量静态受限介电常数)后需要实验验证.

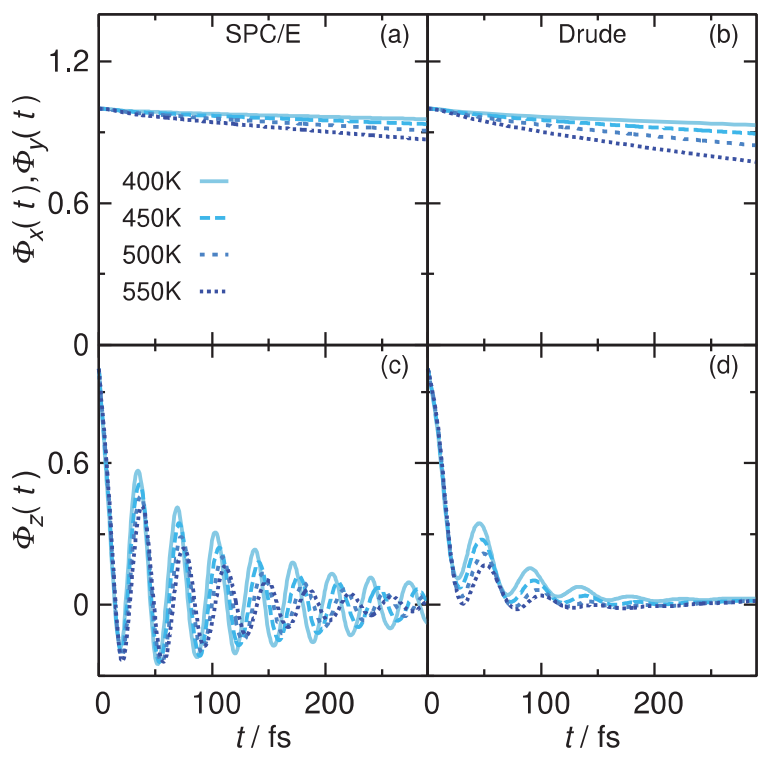

图 7 单分子层受限水偶极矩 (分量) 时间关联函数. (a), (b): $\Phi_{x}(t)$ 或 $\Phi_{y}(t),(\mathrm{c}),(\mathrm{d}): \Phi_{z}(t)$. 左侧 $\mathrm{SPC} / \mathrm{E}$ 水分子模型, 右侧 Drude 极化水分子模型. 不同的线型代表 4 个不同的体系温度

Figure 7 Components of the auto-correlation function. Panel (a), (b): $\Phi_{x}(t)$ or $\Phi_{y}(t)$, panel (c), (d): $\Phi_{z}(t)$. Left column: results of SPC/E model systems, right column: results of SWM4-NDP polarizable model systems. Different line types represent four simulation temperatures

\section{4 结论}

在本工作中, 我们利用平衡态分子动力学的模拟和 受限体系介电性质计算方法, 详细比较两种水分子模型 —经典恒定偶极矩的 $\mathrm{SPC} / \mathrm{E}$ 水分子模型和可极化 Drude 水分子模型, 在描述 $\left(0.65 \mathrm{~nm}\right.$ 限域尺寸、 $5 \times 10^{8} \mathrm{~Pa}$ 限域压强、 4 个不同温度)单分子层受限水和受限冰结构 与介电性质上的优劣势. 统计了 Drude 水模型描述单分 子层受限水和受限冰的水分子偶极矩量值概率密度分
布, 并且计算了每个模拟体系的静态结构因子、静态偶 极空间关联函数、静态介电常数、体系偶极时间关联函 数和德拜弛豫时间. 我们发现了: (1) Drude 极化水分子 模型能够描述出受限水分子极性的奇异特性, 受限冰水结构两相共存的同一温度下，水分子偶极矩平均值不 相等; (2)两种模型描述受限冰结构和受限水空间偶极分 布结构没有明显区别; (3)受限冰的三个分量和受限水 $z$ 分量介电张量数值因水分子转动自由度的晶格和受限 腔约束而被大幅限制; (4)受限水极化弛豫动力学行为也 呈现强烈各向异性. 两种水分子模型体系 $z$ 分量偶极矩 时间关联函数呈周期性振荡衰减结构.

通过本工作系统性比较研究, 我们获得了极化水模 型在受限水体系结构与介电性质描述具体优势较为全 面的认识. 我们首次发现了极化水模型在描述低维度受 限水分子极性特殊变化的绝对性优势, 并提出了极化水 分子模型在受限水介电极化弛豫动力学性质描述上的 潜在优势. 我们也认识到极化水分子模型对于静态介电 常数描述的优势在受限水系统中会因受限条件的增强 而被大幅削减. 因为发现了恒定偶极矩水分子模型和极 化水分子模型在描述受限水和受限冰的静态结构性质 的效果相当, 我们建议受限水物相结构的模拟研究无需 选用极化水分子模型. 综上, 我们认为 Drude 极化水分 子模型在探索受限冰形核形貌、受限水结构相变动力学、 介电性质的调控以及受限体系离子输运溶剂化动力学 过程的模拟研究中是比常用的恒定偶极矩水分子模型 更好的选择.

关于受限水 $z$ 方向介电张量分量物理贡献组成, 本 工作应用的分子模拟技术尚不予以澄清，在未来的工作 中，我们将尝试采用量子 Drude 水分子模型结合能够描 述恒定电势且伴随电荷涨落的受限腔壁 ${ }^{[47]}$ 的分子模拟 技术进一步深入探索. 另外, 我们发现的受限条件下水 分子在冰水两相中极性的跳变现象的物理机理, 也需要 考虑量子效应的影响, 开展独立的研究提供更加充足的 科学证据予以澄清.

\section{References}

[1] Eijkel, J. C. T.; Berg, A. Microfluid Nanofluid 2005, 1, 249.

[2] Granick, S. Science 1991, 253, 1374.

[3] Israelachvili, J. N. Intermolecular and Surface Forces, 3rd ed., Academic Press, Burlington, 2011.

[4] Leikin, S.; Parsegian, V. A.; Rau, D. C.; Rand, R. P. Annu. Rev. Phys Chem. 1993, 44, 369.

[5] Honig, B.; Nicholls, A. Science 1995, 268, 1144.

[6] Cohen-Tanugi, D.; Grossman, J. Nano Lett. 2012, 12, 3602.

[7] Szymczyk, A.; Fatin-Rouge, N.; Fievet, P. J. Colloid Interface Sci. 2007, 309, 245.

[8] Lin, Y.; Shiomi, J.; Maruyama, S.; Amberg, G. Phys. Rev. B 2009 80,045419 .

[9] Mikami, F.; Matsuda, K.; Kataura, H.; Maniwa, Y. ACS Nano 2009 3,1279 .

[10] Toney, M. F.; Howard, J. N.; Richer, J.; Borges, G. L.; Gordon, J. G.; Melroy, O. R.; Wiesler, D. G.; Yee, D.; Sorensen, L. B. Nature 1994, $368,444$.

[11] Ballenegger, V.; Hansen, J. P. J. Chem. Phys. 2005, 122, 114711.

[12] Bonthuis, D. J.; Gekle, S.; Netz, R. R. Phys. Rev. Lett. 2011, 107, 
166102.

[13] Zhang, C.; Gygi, F.; Galli, G. J. Phys. Chem. Lett. 2013, 4, 2477.

[14] Schlaich, A.; Knapp, E. W.; Netz, R. R. Phys. Rev. Lett. 2016, 117, 048001.

[15] Fumagalli, L.; Esfandiar, A.; Fabregas, R.; Hu, S.; Ares, P.; Janardanan, Q. Y.; Radha, B.; Taniguchi, T.; Watanabe, K.; Gomila, G.; Novoselov, K. S.; Geim, A. K. Science 2018, 360, 1339.

[16] Algara-Siller, G.; Lehtinen, O.; Wang, F. C.; Nair, R.; Kaiser, U.; Wu, H.; Geim, A.; Grigorieva, I. Nature 2015, 519, 443.

[17] Du, H.; Liang, H. T.; Yang, Y. Acta Chim. Sinica 2018, 76, 483 (in Chinese). (杜涵, 梁洪涛, 杨洋, 化学学报, 2018, 76, 483.)

[18] Liang, Z.; Du, H.; Liang, H. T.; Yang, Y. Mol. Phys. 2019, 117, 2881.

[19] Vega, C.; Abascal, J. L. F. Phys. Chem. Chem. Phys. 2011, 13, 19663.

[20] Berendsen, H. J. C.; Grigera, J. R.; Straatsma, T. P. J. Phys. Chem 1987, 91, 6269 .

[21] Lamoureux, G.; Harder, E.; Vorobyov, I. V.; Roux, B.; MacKerell, A. D. Chem. Phys. Lett. 2006, 418, 245.

[22] Lybrand, T. P.; Kollman, P. A. J. Chem. Phys. 1985, 83, 2923.

[23] Stuart, S. J.; Berne, B. J. J. Chem. Phys. 1996, 100, 11934.

[24] Dequidt, A.; Devèmy, J.; Pádua, A. A. H. J. Chem. Inf. Model. 2015, $56,260$.

[25] Shepard, A. C.; Beers, Y.; Klein, G. P.; Rothman, L. S. J. Chem. Phys. 1973, 59, 2254.

[26] Gubskaya, A. V.; Kusalik, P. G. J. Chem. Phys. 2002, 117, 5290.

[27] Fernandez, D. P.; Mulev, Y.; Goodwin, A. R. H.; Sengers, J. M. H. L. J. Phys. Chem. Ref. Data 1995, 24, 33.

[28] Jones, A. P.; Crain, J.; Sokhan, V. P.; Whitfield, T. W.; Martyna, G. J. Phys. Rev. B 2013, 87, 144103.

[29] Kimmel, G. A.; Matthiesen, J.; Baer, M.; Mundy, C. J.; Petrik, N. G.; Smith, R. S.; Dohnálek, Z.; Kay, B. D. J. Am. Chem. Soc. 2009, 131, 12838.
[30] Giovambattista, N.; Rossky, P. J.; Debenedetti, P. G. Phys. Rev. Lett. 2009, 102, 050603.

[31] Magda, J. J.; Tirell, M.; Davis, H. T. J. Chem. Phys. 1986, 84, 2901.

[32] Werder, T.; Walther, J. H.; Jaffe, R. L.; Halicioglu, T.; Koumoutsakos, P. J. Phys. Chem. B 2003, 107, 1345.

[33] Hockney, R. W.; Eastwood, J. W. Computer Simulation Using Particles, CRC Press, 1988, p. 55

[34] Yeh, I. C.; Berkowitz, M. J. Chem. Phys. 1999, 111, 3155.

[35] Plimpton, S. J. Comput. Phys. 1995, 117, 1.

[36] Ryckaert, J. P.; Ciccotti, G.; Berendsen, H. J. J. Comput. Phys. 1997, $23,327$.

[37] Jiang, W.; Hardy, D. J.; Phillips, J. C.; MacKerell, A. D.; Schulten, K.; Roux, B. J. Phys. Chem. Lett. 2011, 2, 87.

[38] Wu, A. K.; Lin, S. C.; Karma, A. Phys. Rev. B 2016, 93, 054114.

[39] Luo, C. F.; Fa, W.; Zhou, J.; Dong, J. M.; Zeng, X. C. Nano Lett. 2008, 8, 2607.

[40] Wang, X. H.; Feng, L.; Cao, Z. X. Acta Chim. Sinica 2014, 72, 487 (in Chinese). (王新华, 冯莉, 曹泽星, 化学学报, 2014, 72, 487.)

[41] Zhou, W.; Yin, K. B.; Wang, C. H.; Zhang, Y. Y.; Xu, T.; Borisevich, A.; Sun, L. T.; Idrobo, J. C.; Chisholm, M. F.; Pantelides, S. T.; Klie, R. F.; Lupini, A. R. Nature 2015, 528, E1.

[42] Wang, F. C.; Wu, H. A.; Geim, A. K. Nature 2015, 528, E3.

[43] Chen, J.; Schusteritsch, G.; Pickard, C. J.; Salzmann, C. G.; Michaelides, A. Phys. Rev. Lett. 2016, 116, 025501.

[44] Petrenko, V. F.; Whitworth, R. W. Physics of Ice, Oxford University Press, Oxford, 1999.

[45] Hill, N. E. Trans. Faraday Soc. 1963, 59, 344.

[46] Kindt, J. T.; Schmuttenmaer, C. A. J. Phys. Chem. 1996, 100, 10373.

[47] Yang, P. L.; Wang, Z. X.; Liang, Z.; Liang, H. T.; Yang, Y. Acta Chim. Sinica 2019, 77, 1045 (in Chinese). (杨鹏里, 王振兴, 梁尊, 梁洪涛, 杨洋, 化学学报, 2019, 77, 1045.)

(Cheng, B.) 